homéoprotéines localisées dans les prolongements neuronaux pourraient participer à la régulation de leur guidage. Dans des réseaux matures, elles pourraient moduler la traduction locale dendritique, dont on sait qu'elle joue un rôle crucial dans la plasticité synaptique [10]. $\diamond$ Emx2 in axons: translational functions of homeodomain transcription factors

\section{RÉFÉRENCES}

1. Suda Y, Hossain ZM, Kobayashi C, et al. Emx2 directs the development of diencephalon in cooperation with 0tx2. Development $2001 ; 128: 2433-50$.
2. Hamasaki T, Leingartner A, Ringstedt T, O'Leary DD. EMX2 regulates sizes and positioning of the primary sensory and motor areas in neocortex by direct specification of cortical progenitors. Neuron 2004 ; $43: 359-72$

3. Savaskan NE, Alvarez-Bolado G, Glumm R, et al. Impaired postnatal development of hippocampal neurons and axon projections in the $\varepsilon m \times 2^{-/-}$mutants. J Neurochem 2002 ; 83 : 1196-207.

4. Galli R, Fiocco R, De Filippis $L$, et al. Emx2 regulates the proliferation of stem cells of the adult mammalian central nervous system. Development 2002 ; 129 : 1633-44.

5. Nedelec S, Foucher I, Brunet I, et al. Em×2 homeodomain transcription factor interacts with eukaryotic translation initiation factor $4 \varepsilon$ (elF4E) in the axons of olfactory sensory neurons. Proc Natl Acad Sci USA 2004 ; 101 : 10815-20.
6. Campbell DS, Holt CE. Chemotropic responses of retinal growth cones mediated by rapid local protein synthesis and degradation. Neuron 2001 ; 32 : 1013-26.

7. Feinstein P, Bozza T, Rodriguez I, et al. Axon guidance of mouse olfactory sensory neurons by odorant receptors and the $\beta 2$ adrenergic receptor. Cell 2004 ; $117: 833-46$.

8. Topisirovic I, Culjkovic B, Cohen N, et al. The prolinerich homeodomain protein, $\mathrm{PRH}$, is a tissue-specific inhibitor of elF4E-dependent cyclin Dl mRNA transport and growth. EMBO J $2003 ; 22$ : 689-703.

9. Niessing D, Blanke $S$, Jackle H. Bicoid associates with the 5'-cap-bound complex of caudal mRNA and represses translation. Genes Dev 2002 ; 16 : 2576-82.

10. Kelleher RJ 3rd, Govindarajan A, Tonegawa S. Translational regulatory mechanisms in persistent forms of synaptic plasticity. Neuron 2004; 44 : 59-73.

NOUVELLE

\title{
Rôles de PPAR $\delta$ dans la physiologie du muscle squelettique
}

Paul A. Grimaldi

> Les peroxisome proliferator-activated receptors (PPAR) sont des facteurs de transcription activés par les acides gras et certains de leurs métabolites. Ils participent à la régulation du métabolisme et au contrôle de la différenciation de plusieurs tissus. Les isoformes $\alpha$ et $\gamma$ ont été les plus étudiées, en particulier parce qu'elles sont les relais des effets de molécules hypolipémiantes (fibrates) ou antidiabétiques (thiazolidinediones). Récemment, il a été établi que PPAR $\delta$ était également une cible thérapeutique intéressante pour le traitement du syndrome métabolique. Ainsi, chez le singe et la souris obèses, le traitement par un agoniste PPAR $\delta$ normalise le profil lipidique, améliore la réponse à l'insuline et diminue l'obésité $[1,2]$.

Ces deux dernières années, il a été montré que le muscle squelettique était une cible privilégiée des agonistes de PPAR $\delta$. $\varepsilon n$ effet, des arguments pharmacologiques et génétiques ont prouvé que PPAR $\delta$ joue un rôle central dans la régu- lation du catabolisme des acides gras dans les cellules musculaires en culture. Dans les myotubes $\mathrm{C} 2 \mathrm{C} 12$ ou L6, PPAR $\delta$ contrôle directement l'expression de la plupart des protéines impliquées dans le catabolisme des lipides, et son activation conduit à l'augmentation de la $\beta$ oxydation des acides gras [2, 3].

Des études réalisées in vivo, grâce à des modèles de souris transgéniques qui présentent une surexpression de PPAR $\delta$ spécifique dans le muscle squelettique, ont révélé une fonction inattendue pour PPAR $\delta$ dans la physiologie musculaire. La première publication a montré que cette augmentation de l'expression de PPAR $\delta$ conduisait à des changements profonds de la composition en fibres des différents muscles, avec une augmentation du nombre de fibres de type oxydatif (augmentation de 2,7 fois dans le muscle tibialis anterior), alors que le nombre de fibres glycolytiques ne varie pas. Ce remodelage musculaire conduit à une augmentation des capacités oxyda-
Inserm U.636, Centre de Biochimie, Parc Valrose, UNSA, 06108 Nice, France. grimaldi@unice.fr

tives musculaires et donc du catabolisme des acides gras. Ce nouveau phénotype musculaire s'accompagne d'une très nette réduction de la masse grasse due à une diminution de la taille moyenne des adipocytes [4]. Ces observations ont été confirmées très récemment par un autre groupe qui montre, en outre, que la surexpression musculaire de PPAR $\delta$ confère une protection très efficace contre les effets néfastes des régimes riches en lipides, comme l'obésité et la résistance à l'insuline, et augmente la résistance à la fatigue musculaire [5]. Ainsi, les animaux qui surexpriment PPAR $\delta$ dans leurs muscles acquièrent un phénotype musculaire et métabolique similaire au phénotype «marathonien » induit par l'entraînement d'endurance de longue durée [6]. Cela est tout à fait inattendu, puisque ces animaux ont une activité physique qui n'est pas perturbée. Ces observations suggèrent que PPAR $\delta$ est un acteur important dans le remodelage muscu- 
laire induit par l'exercice physique. La démonstration que l'expression de PPAR $\delta$ est augmentée d'un facteur 3, environ, dans les muscles d'animaux témoins soumis à un exercice modéré (45 minutes de natation par jour pendant 3 semaines) est un argument fort en faveur de cette hypothèse [4].

L'ensemble de ces données indique donc que PPAR $\delta$ joue un rôle important dans la physiologie musculaire. D'une part, son activation par des agonistes synthétiques conduit à une augmentation de l'expression des protéines impliquées dans le catabolisme des acides gras. D'autre part, le facteur de transcription joue probablement un rôle majeur dans les mécanismes d'adaptation du muscle à l'entraînement à l'exercice d'endurance, qui se traduit par une augmentation de la capacité intrinsèque du muscle à consommer des acides gras.

La découverte de ces nouvelles fonctions de PPAR $\delta$ dans le muscle permet d'apporter un début d'explication aux effets bénéfiques de ces agonistes synthétiques chez les animaux obèses. II peut être proposé que, comme le fait l'exercice physique d'endurance, la suractivité de PPAR $\delta$ augmente la consommation de lipides dans le muscle sque- lettique et diminue la disponibilité en acides gras pour la synthèse de triglycérides dans le tissu adipeux, ce qui provoque une réduction de l'adiposité et une normalisation de la production d'adipocytokines (Figure 1). Ces observations font de l'utilisation d'agonistes de PPAR $\delta$ une approche thérapeutique nouvelle pour le traitement du syndrome métabolique. Toutefois, le récepteur nucléaire a un profil d'expression tissulaire large et, afin d'éviter des effets néfastes de traitements par de telles molécules, il devient urgent de connaître les fonctions de PPAR $\delta$ dans d'autres tissus, comme l'intestin ou le cœur. $\diamond$

Roles of PPAR $\delta$ in skeletal muscle physiology

\section{RÉFÉRENCES}

1. Oliver WR, Shenk JL, Snaith MR, et al. A selective peroxisome proliferator-activated receptor $\delta$ agonist promotes reverse cholesterol transport. Proc Natl Acad Sci USA 2001; 98: 5306-11.

2. Tanaka T, Yamamoto J, Iwasaki $S$, et al. Activation of peroxisome proliferator-activated receptor $\delta$ induces fatty acid $\beta$-oxidation in skeletal muscle and attenuates metabolic syndrome. Proc Natl Acad Sci USA 2003; 100: 15924-9.

3. Holst D, Luquet $S$, Nogueira V, et al. Nutritional regulation and role of peroxisome proliferatoractivated receptor $\delta$ in fatty acid catabolism in skeletal muscle. Biochim Biophys Acta 2003; 1633: 43-50.

4. Luquet S, Lopez-Soriano J, Holst D, et al. Peroxisome proliferator-activated receptor $\delta$ controls muscle development and oxidative capabilities. FASEB J 2003; 17: 2299-301.

5. Wang $Y X$, Zhang $C L, y u R T$, et al. Regulation of muscle fiber type and running endurance by PPAR $\delta$. PLOS Biol $2004 ; 2: 1-8$.

6. Allen DL, Harrison BC, Maass A, et al. Cardiac and skeletal muscle adaptations to volontary running in the mouse. J Appl Physiol 2001; 90:1900-8.

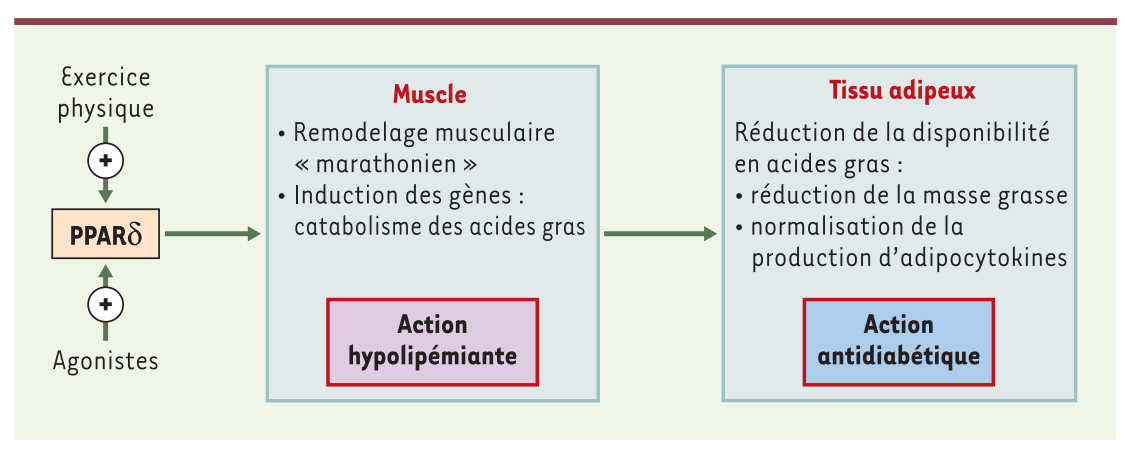

Figure 1. Activation de PPAR $\delta$ dans le muscle et correction du syndrome métabolique.

\section{NOUVELlE}

\section{Un nouveau rôle pour le récepteur périphérique des benzodiazépines?}

Mariano A. Ostuni, Jean-Jacques Lacapère

\begin{abstract}
Inserm U.683, Faculté de Médecine Xavier Bichat, 16, rue Henri Huchard, 75870 Paris Cedex 18, France.
\end{abstract}

lacapere@bichat.inserm.fr isoquinoline, le PK 11195, a été décrite comme le ligand le plus spécifique du PBR. Les études de fractionnement subcellulaire ont permis de montrer que le PBR présentait une localisation majoritairement mitochondriale, avec une insertion dans la
> Le récepteur périphérique des benzodiazépines (PBR) est une protéine membranaire de $18 \mathrm{kDa}$ initialement mise en évidence par ses propriétés de liaison du diazépam (Valium ${ }^{\circledR}$ ) dans les tissus n'appartenant pas au système nerveux central. Cette localisation s'accompagne de propriétés pharmacologiques qui le distinguent du récepteur central des benzodiazépines, encore appelé récepteur GABAa. En particulier, le PBR présente une affinité plus faible pour le diazépam que le récepteur central. Plusieurs classes de ligands ont été testées et une membrane externe de cet organite. Le PBR est exprimé dans tous les tissus examinés à ce jour, la plus forte expression étant observée dans les tissus connus pour synthétiser les hormones stérö̈diennes, glandes surrénales, testicules, ovaires, et dans les astrocytes [1]. 